DOI: https://doi.org/10.46296/ig.v5i9edespfeb.0055

\title{
COMBINACIÓN DE MEZCLAS DE AGREGADOS GRUESOS Y FINOS PERTENECIENTES A LA CANTERA URUZCA PARA DIFERENTES RESISTENCIAS DE HORMIGÓN
}

\section{COMBINATION OF MIXTURES OF COARSE AND FINE AGGREGATES FROM THE URUZCA'S QUARRY FOR DIFFERENT CONCRETE STRENGTHS}

\author{
Cedeño-Cedeño Héctor Eduardo ${ }^{\text {; }}$ Chávez-Chon Long Jaime Emilio ${ }^{2}$; \\ Macías-Sánchez Lucia Katherine ${ }^{3}$; Ortiz-Hernández Eduardo Humberto ${ }^{4}$ \\ 1 Universidad Técnica de Manabí. Portoviejo, Ecuador. Correo: hcedeno2410@utm.edu.ec. \\ 2 Universidad Técnica de Manabí. Portoviejo, Ecuador. Correo: Ichavez9946@utm.edu.ec. \\ 3 Universidad Técnica de Manabí. Portoviejo, Ecuador. Correo: lucia.macias@utm.edu.ec. \\ ${ }^{4}$ Universidad Técnica de Manabí. Portoviejo, Ecuador. Department of Civil Engineering, \\ University of Alicante, 03690. Alicante, Spain. Correo: eduardo.ortiz@utm.edu.ec.
}

\begin{abstract}
Resumen
Esta investigación se enfocó en diseñar una mezcla de agregados pétreos para posterior ser utilizados en diseño de hormigones de $210 \mathrm{~kg} / \mathrm{cm}^{2}$ y $240 \mathrm{~kg} / \mathrm{cm}^{2}$. Para esta mezcla se recopilaron materiales de la cantera Uruzca a los que se les realizaron ensayos granulométricos, para realizar la mezcla de agregados se empleó un método de tanteo; se realizaron ensayos complementarios de peso específico, peso unitario compactado y suelto y porcentaje de humedad necesarios para el Método $\mathrm{ACl}$ 211.1. Las proporciones de materiales finos que fueron seleccionadas corresponden a un porcentaje de $80 / 20$ (80\% cisco y $20 \%$ arena de banco) con lo que se mejoró su graduación y el módulo de finura se ajustó al rango de 2.3 a 3.1, las resistencias a la compresión fueron superadas pero la resistividad eléctrica demostró alta penetración de cloruros, dejando marcado una durabilidad deficiente en ambientes de alta contaminación. Las proporciones de materiales que se requieren para diseñar un hormigón de $210 \mathrm{~kg} / \mathrm{cm}^{2}$ son de 1 de cemento con 4.36 de agregado grueso y fino, mientras que para una mezcla de $240 \mathrm{~kg} / \mathrm{cm}^{2}$ son 1 de cemento y 3.85 de agregado grueso y fino.
\end{abstract}

Palabras clave: Hormigón, agregados grueso, agregado fino, resistividad eléctrica, resistencia.

\begin{abstract}
This research focused on designing a mixture of stone aggregates to be used in the design of concrete of $210 \mathrm{~kg} / \mathrm{cm}^{2}$ and $240 \mathrm{~kg} / \mathrm{cm}^{2}$. For this mix, materials were collected from the Uruzca quarry and granulometric tests were carried out on them. To make the aggregate mix, a trial and error method was used; complementary tests of specific weight, compacted and loose unit weight and percentage of moisture required by the $\mathrm{ACl} 211.1$ Method were carried out. The proportions of fine materials that were selected correspond to a percentage of $80 / 20(80 \%$ cisco and $20 \%$ bench sand) which improved its grading and the fineness modulus was adjusted to the range of 2.3 to 3.1, the compressive strengths were exceeded but the electrical resistivity showed high penetration of chlorides, leaving a deficient durability in high contamination environments. The proportions of materials required to design a $210 \mathrm{~kg} / \mathrm{cm}^{2}$ concrete are 1 of cement with 4.36 of coarse and fine aggregate, while for a $240 \mathrm{~kg} / \mathrm{cm}^{2}$ mix they are 1 of cement and 3.85 of coarse and fine aggregate.
\end{abstract}

Keywords: Concrete, coarse aggregate, fine aggregate, electrical resistivity, strength.

Información del manuscrito:

Fecha de recepción: 21 de diciembre de 2021.

Fecha de aceptación: 01 de febrero de 2022.

Fecha de publicación: 07 de febrero de 2022. 


\section{Introducción}

Los agregados son una parte indispensable en la composición del hormigón. Actualmente son muchas las normativas y estándares internacionales que se han creado, con la finalidad de que la producción de estos materiales sea la más adecuada y sobre todo que permitan obtener un resultado óptimo al momento de crear hormigones de diferentes resistencias.

A pesar de que la producción de estos materiales en nuestro medio no se detiene, los yacimientos de estos si son finitos y tal como expone (Soto \& Escobedo, 2006) la búsqueda de alternativas viables para el diseño de hormigones es algo que debe ser una prioridad para las nuevas generaciones de ingenieros.

Bajo estos contextos se han librado ciertas investigaciones que han empleado materiales como arenas de ríos (Panchana et al., 2020), en post de buscar alternativas para crear hormigones, debido a que la constante producción de estos genera cambios en los ecosistemas que muchas veces son permanentes. También se ha creado una mezcla de materiales que se comercializan bajo el nombre de "rocarena" para diferentes tipos de hormigones, esto como parte de una propuesta de mejorar la calidad de los procesos constructivos en nuestro medio.

Con la presente investigación se busca establecer una mezcla de agregados pétreos con los que se pueda estandarizar una combinación de hormigones para resistencias de $210 \mathrm{~kg} / \mathrm{cm} 2$ y $240 \mathrm{~kg} / \mathrm{cm} 2$, el proceso de esta mezcla de agregados será llevada a cabo empleando el porcentaje de material pasante; a esto se espera que con el cumplimiento de la normativa ecuatoriana se obtengan un material que cumpla con las fajas granulométricas y que su vez la resistencia a la compresión alcance - sobrepase la resistencias de diseño.

Lo que se busca con la creación de esta mezcla es tener materiales que sean fáciles de manipular, y que a su vez logren alcanzar los estándares de las normativas que rigen los procesos constructivos en el Ecuador. Esto como parte de un proceso de avance hacia estrategias constructivas más amigables con el medio ambiente. Este tipo de 
trabajos a su vez busca que la manipulación de materiales en obra sea más eficiente y que la calidad del hormigón se vea lo menos afectada posible. La finalidad de los ensayos es determinar los parámetros físicos y mecánicos de los agregados gruesos y finos (Hernández, E. H. O., Vínces, J. J. G., Castro, C. M. J., \& Zambrano, R. V. H., 2020), realizados bajo Norma Técnica Ecuatoriana.

\section{Metodología}

\section{A. Materiales.}

Bajo la premisa de crear una mezcla de agregados que permitan obtener una resistencia de $210 \mathrm{~kg} / \mathrm{cm}^{2}$ y $240 \mathrm{~kg} / \mathrm{cm}^{2}$ se buscó materiales pétreos procedentes del cantón Portoviejo, provincia de Manabí. Se utilizo un Cemento Portland Tipo GU procedente de la empresa HOLCIM.

\section{Cemento Portland Tipo GU.}

Este en un material fino, con una coloración grisácea que surge de la trituración de piedras calizas y arcillosas que son los elementos básicos para formar el Clinker. La formación del Clinker tal como lo indica (Romo, 2008, p. 4) es producto de la calcinación de estos elementos a temperaturas de $\pm 1400^{\circ} \mathrm{C}$. En este proceso se pueden añadir elementos como "agua, sulfatos de calcios, piedras calizas hasta un 5\%" (NTE INEN 151, 2010).

En base a la clasificación de cementos que se indican en (NTE INEN 2 380, 2005) y tal como lo especifica el fabricante en el cumplimiento (NTE INEN 156, 2009) la densidad del cemento empleada es de $3.15 \mathrm{~g} / \mathrm{cm} 3$ o $3150 \mathrm{~kg} / \mathrm{cm} 3$ para los respectivos cálculos de dosificación.

\section{Agregado grueso pasante $3 / 4$ in.}

El origen de este material es de la Cantera URUZCA concesión Rio de Oro. Autores como (McCormac \& Brown, 2011, pp. 18-19; Romo, 2008, p. 6) describen que un agregado triturado o producido por algún método similar presenta mejores características de adherencia por tener aristas. El material empleado en su totalidad ha sido "retenido por el tamiz $\mathrm{N}^{\circ} 4$ " (NTE INEN 694, 2010, pp. 2-3). 
De los ensayos granulométricos realizados se obtuvieron los siguientes resultados:

- Módulo de finura (NTE INEN 694, 2010; NTE INEN 696, 2010): 2.62

- Humedad (NTE INEN 862, 2011, pp. $2-3): 0.23 \%$

- Peso Unitario Suelto (PUS) (NTE INEN 858, 2010): 1489.13 $\mathrm{kg} / \mathrm{cm}^{3}$

- Peso Unitario Compactado (PUC) (NTE INEN 858, 2010): $1566.64 \mathrm{~kg} / \mathrm{cm}^{3}$

- Gravedad específica de masa (NTE INEN 856, 2010): 2578.95 $\mathrm{kg} / \mathrm{cm} 3$

- Gravedad específica de Saturado Superficialmente Seco (S.S.S) (NTE INEN 856, 2010): $2631.58 \mathrm{~kg} / \mathrm{cm}^{3}$

- Absorción (NTE INEN 856, 2010): $2.04 \%$.

\section{Agregado fino.}

Para el presente trabajo se utilizaron dos tipos de áridos finos basados con la norma (NTE INEN 694, 2010, p. 2). El primero en utilizar fue el agregado fino (Cisco) proveniente de la cantera Uruzca, y el otro fue una arena de banco extraída del sector Los Tamarindos (553226.70 m E;
$9905662.11 \mathrm{~m} \mathrm{~S}$ ) de la parroquia Crucita del cantón Portoviejo.

\subsection{Cisco.}

Aplicando las respectivas normativas para determinar las propiedades del cisco se obtuvieron los siguientes resultados:

- Módulo de finura (NTE INEN 694, 2010; NTE INEN 696, 2010): 3.32

- Peso Unitario Suelto (PUS) (NTE INEN 858, 2010): 1650.24 $\mathrm{kg} / \mathrm{cm} 3$

- Peso unitario compactado (PUC) (NTE INEN 858, 2010): 1792.83 $\mathrm{kg} / \mathrm{cm} 3$

- Gravedad específica de masa (NTE INEN 856, 2010): 2702.14 $\mathrm{kg} / \mathrm{cm} 3$

- Gravedad especifica de S.S.S. (NTE INEN 856, 2010): 2704.35 $\mathrm{kg} / \mathrm{cm} 3$

- Absorción (NTE INEN 856, 2010): $0.082 \%$

\subsection{Arena.}

La arena fue obtenida del sitio Los Tamarindos (553226.70 m E; $9905662.11 \mathrm{~m} \mathrm{~S})$ de la parroquia Crucita del cantón Portoviejo. Esta se analizó aplicando los criterios de la normativa ecuatoriana y se 
obtuvieron las siguientes

propiedades:

- Módulo de finura (NTE INEN 694, 2010; NTE INEN 696, 2010): 0.90

- Peso Unitario Suelto (PUS) (NTE INEN 858, 2010): 1431.52 $\mathrm{kg} / \mathrm{cm}^{3}$

- Peso Unitario Compactado (PUC) (NTE INEN 858, 2010):1594.42 kg/cm ${ }^{3}$

- Gravedad específica de masa (NTE INEN 856, 2010): 2661.33 $\mathrm{kg} / \mathrm{cm} 3$

- Gravedad específica de S.S.S. (NTE INEN 857, 2010): 2686.20 $\mathrm{kg} / \mathrm{cm}^{3}$

- Absorción (NTE INEN 857, 2010): $0.93 \%$

\section{Agua.}

Bajo el criterio de la norma (NTE INEN 2617, 2012, p. 2) en su literal 5.1.1.2 se deja en claro que se puede emplear agua potabilizada para el amasado de hormigón de cemento hidráulico. La cual fue empleada para el diseño de la presente investigación

\section{Aditivo.}

Este es un aditivo superplastificante "reductor de agua de alto rango"
(EUCOMEX, 2020). El empleo de este permite que la dispersión de partículas de agua sea más eficiente logrando de esta forma una mejor hidratación del cemento y por ende una menor cantidad de agua durante el amasado.

La denominación comercial de este aditivo es "EUCON 37" (EUCOMEX, 2020), que posee una composición química de elementos sintéticos con una densidad de $1.20 \pm 0.024 \mathrm{~g} / \mathrm{ml}$.

\section{B. Métodos.}

Fase 1: Recolección de muestras.

La procedencia de los materiales a utilizarse fue (pasante $3 / 4$ in $y$ el Cisco) que son de la Cantera URUZCA concesión Rio de Oro. Por su parte la arena de banco fue obtenida en el sitio Los Tamarindos (553226.70 m E; $9905662.11 \mathrm{~m} \mathrm{~S}$ ) de la parroquia Crucita. Ambos materiales son procedentes del cantón Portoviejo.

Fase 2: Ensayos de laboratorio.

Lo primero que se realizó fueron los ensayos de Granulometría (NTE INEN 694, 2010; NTE INEN 696, 2010), Pesos Unitarios Sueltos (PUS) y Compactados (PUC) (NTE 
INEN 858, 2010), Gravedad especifica de masa y Gravedad especifica de superficie saturada seca S.S.S, Porcentaje de absorción (NTE INEN 857, 2010) y Porcentaje de humedad (NTE INEN 862, 2011).

Para el caso de los agregados finos se tamizo la $3 / 8$ in con la finalidad de eliminar cualquier impureza. En el cisco se observó la presencia de material fino, por lo cual se procedió a realizar el ensayo de Límite Plástico (LP) (NTE INEN 692, 1982) una vez obtenido la plasticidad del material se tomó la decisión de remover el material pasante del tamiz $N^{\circ} 200$ por medio lavado y posterior secado.

Fase 3: Diseño de mezcla.

Para realizar la combinación de agregados se usaron las granulometrías de los áridos finos (cisco y arena) y se empleó el porcentaje pasante. Los límites inferior y superior empleados para el análisis de la mezcla de agregados fueron los proporcionados por (MOP, 2002, p. VIII 25-36; NTE INEN 872, 2011, pp. 2-5).

Fase 4: Ensayos de resistencia a la compresión y resistividad.
Bajo la meta de elaborar una mezcla de agregados en los que se puedan diseñar hormigones de resistencias de $210 \mathrm{~kg} / \mathrm{cm} 2$ y $240 \mathrm{~kg} / \mathrm{cm} 2$, el diseño del hormigón fue realizado empleando la metodología del $\mathrm{ACl}$ 211.1 y las consideraciones de (Quiroz \& Salamanca, 2006, pp. 163172; Zabaleta, 1992, pp. 81-94).

Los ensayos de resistencia a la compresión se realizaron a los 7, 14 y 28 días considerando los criterios de ruptura que se indican en la (NTE INEN 1573, 2010). Para el ensayo de la resistividad eléctrica se aplicó el criterio de la normativa ASTM C1202 enfocada al ensayo de penetración de cloruros.

\section{Resultados}

\section{a) Diseño de la mezcla de agregados finos.}

La investigación se enfoca en la creación de una mezcla de agregados que permitan tener una mejor optimización de materiales, diseños estandarizados de hormigón y sobre todo que esta cumpla con los requisitos mínimos de resistencias a la compresión de $210 \mathrm{~kg} / \mathrm{cm}^{2}$ y $240 \mathrm{~kg} / \mathrm{cm}^{2}$. 
Lo primero que se obtuvo en los agregados grueso y finos son las propiedades físicas y mecánicas para realizar la mezcla de materiales como (cisco, arena y grava). Se aplicaron las normativas NTE INEN relacionadas a los áridos y criterios de diseños de hormigones. La mezcla como tal se realizó aplicando una metodología de tanteo como se explica en el trabajo de (Montejo et al., 2018, pp. 35-47).

Aplicando los criterios de la norma (NTE INEN 696, 2010) se obtuvieron los siguientes valores de porcentaje pasante de estos materiales.

Tabla 1. Porcentaje pasante del cisco lavado y la arena.

\begin{tabular}{|c|c|c|}
\hline$\overline{\text { TAMIZ }}$ & ARENA \% & CISCO \% \\
\hline $3 / 8^{\prime \prime}$ & 100.00 & 100.00 \\
\hline$N^{\circ} 4$ & 100.00 & 94.00 \\
\hline$N^{\circ} 8$ & 100.00 & 67.93 \\
\hline$N^{\circ} 16$ & 100.00 & 49.18 \\
\hline$N^{\circ} 30$ & 99.99 & 34.78 \\
\hline$N^{\circ} 50$ & 98.85 & 19.58 \\
\hline$N^{\circ} 100$ & 11.23 & 7.35 \\
\hline
\end{tabular}

Comparando estos valores con los límites máximos y mínimos que están indicados por (MOP, 2002, p. VIII 25-36; NTE INEN 872, 2011, pp. 2-5). Se observa que el cisco cumple con las especificaciones necesarias para considerarlo como un material graduado correctamente. Para el caso de la arena no sucede lo mismo, se observa claramente que este no cumple con los criterios necesarios para catalogarla como un material bien graduado; en cierta parte se debe a que esta arena es natural o conocida en el medio como arena de banco, además esta posee un módulo de finura de 0.90 en donde indica que sus partículas son muy finas.

Un punto de partida para considerar realizar la mezcla de agregados se basa en el criterio expuesto por (MOP, 2002, p. VIII 25-36), (Kosmatka \& Wilson, 2011, p. 101) y la norma ASTM C33, en donde se menciona que el árido fino no debe tener un módulo de finura mayor a 3.1 y tampoco menor a 2.3 , por lo que en los ensayos granulométricos realizados al cisco se obtuvo un módulo de finura de 3.27 , lo cual supera el valor recomendado.

Con el método de tanteo, se obtuvo que la relación más óptima para combinar ambos materiales finos será de un $80 \%$ de cisco con un $20 \%$ arena. Dando como resultado la siguiente gráfica №2 ilustrada: 
Grafica 2: Resultados de la combinación de $80 \%$ cisco y $20 \%$ arena. La línea roja indica el comportamiento de la mezcla.

\section{Granulometría de la combinacion $80 / 20$}

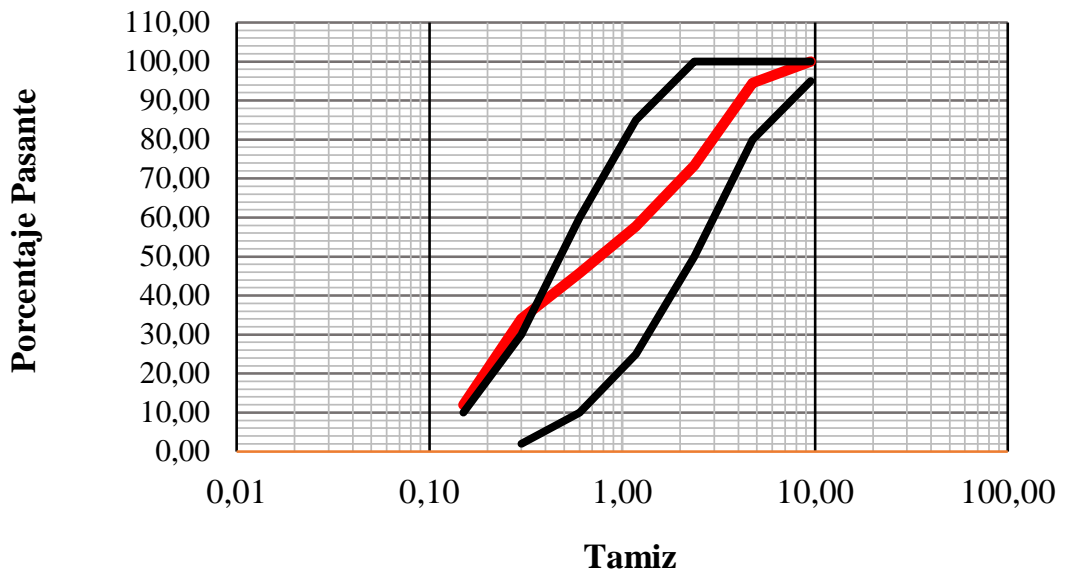

La gráfica muestra el no homogénea 0 comúnmente comportamiento de la combinación conocidos como hormigueros.

de agregados finos, ajustada con $80 \%-20 \%$ en donde indica que en parte superior de estos agregados cruza los límites expuestos por el (MOP, 2002, p. VIII 25-36; NTE INEN 872, 2011, pp. 2-5). En este mismo caso se pudo obtener un módulo de finura con la misma mezcla el cual fue de 2.83 el cual no indica que está dentro de los rangos recomendados.

Con la mezcla lo que se espera es cubrir todos los espacios posibles durante el amasado del hormigón. Y tal como se menciona en (Parker \& Ambrose, 2008, p. 31) una correcta distribución permite que se cubran todos los espacios entre los agregados bien graduados, evitando de esta forma la creación de mezcla

Para diseñar la mezcla de hormigón empleando el método del $\mathrm{ACl} 211.1$ fue necesario realizarle a la combinación de agregados los siguientes ensayos obteniendo los siguientes resultados:

- Módulo de finura (NTE INEN 694, 2010; NTE INEN 696, 2010): 2.83

- Peso Unitario Suelto (PUS) (NTE INEN 858, 2010): 1769.33 $\mathrm{kg} / \mathrm{cm}^{3}$

- Peso Unitario Compactado (PUC) (NTE INEN 858, 2010): $1918.18 \mathrm{~kg} / \mathrm{cm}^{3}$

- Gravedad especifica de masa (NTE INEN 856, 2010) : 2564.79 $\mathrm{kg} / \mathrm{cm}^{3}$ 
- Gravedad especifica de S.S.S. (NTE INEN 857, 2010): 2619.06 $\mathrm{kg} / \mathrm{cm}^{3}$

- Absorción (NTE INEN 857, 2010): $2.12 \%$

- Humedad (NTE INEN 862, 2011, pp. $2-3): 0.26 \%$

Basado en estos valores se procedió a comenzar con el diseño de las mezclas de hormigón de acuerdo al criterio del método del $\mathrm{ACl} 211.1$.

\section{b) Diseños de mezclas para hormigones.}

El método de diseño de mezclas de concreto del $\mathrm{ACl} 211.1$ es uno de los muchos métodos de diseño de hormigones que existen a nivel mundial, aunque en nuestro medio es el más popularizado y se ha visto aplicado en diversas investigaciones como las de (Alvarado et al., 2019; Panchana et al., 2020).

Basándose en la metodología de cálculo y con los datos obtenidos de los áridos, se obtuvieron los siguientes requerimientos de cemento y agregados para un diseño de hormigón de $210 \mathrm{Kg} / \mathrm{cm}^{2}$ y 240 $\mathrm{Kg} / \mathrm{cm}^{2}$.
Tabla 2. Diseño de Hormigón de 210 $\mathrm{Kg} / \mathrm{cm}^{2}$, las proporciones están calculadas en base al cemento.

\begin{tabular}{ccc}
\hline Elemento & Cantidad & $\begin{array}{c}\text { Proporción } \\
\mathbf{m}^{\mathbf{3}}\end{array}$ \\
\hline Cemento & $377.62 \mathrm{Kg}$ & 1.00 \\
Ripio & $789.01 \mathrm{Kg}$ & 2.09 \\
$\begin{array}{c}\text { Combinación } \\
80 / 20\end{array}$ & $\begin{array}{c}858.92 \mathrm{Kg} \\
\text { Agua }\end{array}$ & $\begin{array}{c}246.22 \\
\text { Litros }\end{array}$ \\
\hline
\end{tabular}

Tabla 3. Diseño de Hormigón de 240 $\mathrm{Kg} / \mathrm{cm}^{2}$, las proporciones están calculadas en base al cemento.

\begin{tabular}{ccc}
\hline Elemento & Cantidad & $\begin{array}{c}\text { Proporción } \\
\mathbf{m}^{\mathbf{3}}\end{array}$ \\
\hline Cemento & $418.60 \mathrm{Kg}$ & 1.00 \\
Ripio & $755.55 \mathrm{Kg}$ & 1.80 \\
Combinación & & \\
$80 / 20$ & $858.92 \mathrm{Kg}$ & 2.05 \\
& 245.60 & \\
Agua & Litros & 0.59 \\
\hline
\end{tabular}

Evaluando las mezclas de agregados pétreos se obtuvieron los siguientes resultados:

- Para un diseño de hormigones de $210 \mathrm{~kg} / \mathrm{cm}^{2}$ la relación a trabajarse sería 1 de cemento con 4.36 de agregados grueso finos (2.09 de grava pasante tamiz $3 / 4$ in y 2.27 de combinación entre cisco/arena).

- En el caso hormigones de 240 $\mathrm{kg} / \mathrm{cm}^{2}$ la relación a trabajarse sería 1 de cemento con 3.85 de agregados grueso y finos (1.80 de grava pasante tamiz $3 / 4$ in $y$ 
2.05 de combinación entre cisco/arena).

Los moldes empleados para la elaboración de los cilindros poseen dimensiones de $10 \mathrm{~cm}$ de diámetro por $20 \mathrm{~cm}$ de altura, cumplimiento con los requerimientos que se estipulan en (NTE INEN 1573, 2010).

El llenado de estos fue realizado con la ayuda de un cucharon y con una varilla de punta redondeada en tres capaz de 25 golpes tal como se indica la norma INEN 1573.

El control de la trabajabilidad del hormigón se realizó usando el ensayo del Cono de Abrams o también conocida como prueba de revenimiento, esto bajo el criterio de la (NTE INEN 1578, 2010). Para el caso de este diseño se empleó un revenimiento de 4 pulgadas como se ilustra en la gráfica 3.

Grafica 3: Revenimiento en hormigón 210 $\mathrm{kg} / \mathrm{cm}^{2}(A)$, y revenimiento para hormigón de $240 \mathrm{~kg} / \mathrm{cm}^{2}(B)$.

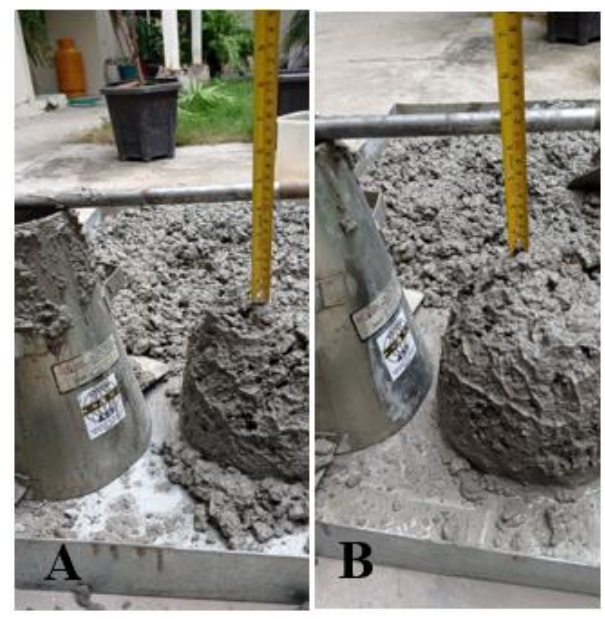

En

algunas

normativas

internacionales se menciona que un revenimiento de 10 - $15 \mathrm{~cm}$ (4 a 6 in) en el hormigón es sinónimo de una consistencia fluida.

Como parte del proceso de curado de las probetas creadas, estas desde el momento de la remoción del molde y su posterior rotulación fueron sumergidas en una piscina con agua hasta sus respectivos días de ensayo a la compresión y resistividad eléctrica.

\section{c) Resistencia a la compresión.}

Este parámetro fue realizado utilizando las indicaciones expuestas por la normativa (NTE INEN 1573, 2010). Los especímenes creados se ensayaron en una frecuencia de ruptura de 7, 14 y 28 días, siendo este último día el de principal interés, porque en este tiempo es donde se logra obtener parte de la máxima resistencia del hormigón como e ilustra en la gráfica N4 la resistencia a la compresión de las probetas de $210 \mathrm{~kg} / \mathrm{cm}^{2}$ y $240 \mathrm{~kg} / \mathrm{cm}^{2}$. 
Grafica 4: Resistencia a la compresión a los 7, 14 y 28 días de elaboración de las probetas.

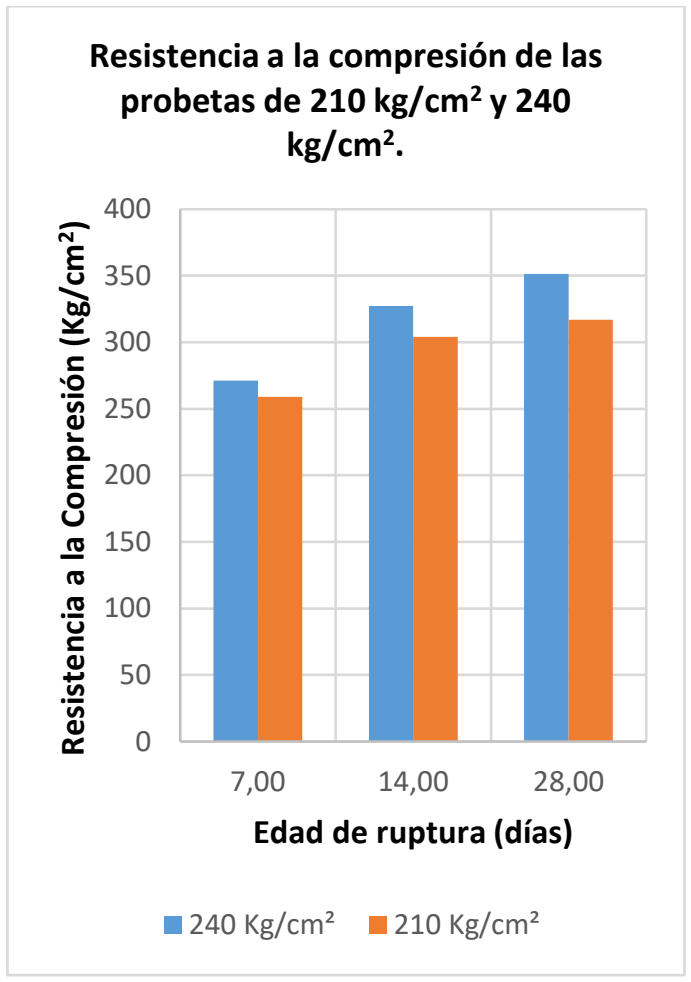

Como se puede observar en los resultados, el objetivo de obtener hormigones de $210 \mathrm{~kg} / \mathrm{cm} 2$ y 240 $\mathrm{kg} / \mathrm{cm} 2$ ha sido alcanzado, pero se deben tener en consideraciones ciertos aspectos como la durabilidad y la eficiencia del comportamiento ante agentes químicos (sulfatos, cloruros y otros). Este último permite comprender que tan durable será el hormigón diseñado cuando sea expuesto al ambiente y a todos los agentes químicos que existan en él.
Grafica 5: Ensayo de compresión axial en hormigón de $210 \mathrm{~kg} / \mathrm{cm}^{2}(A)$, y hormigón de $240 \mathrm{~kg} / \mathrm{cm}^{2}(B)$.
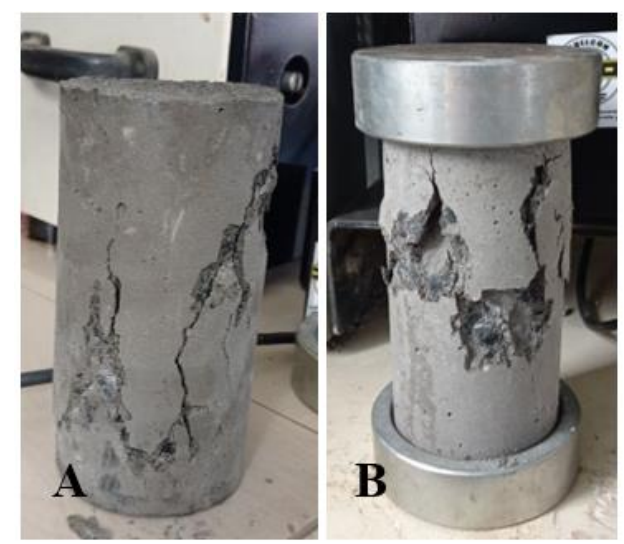

Al momento de realizar los ensayos a compresión, el agregado grueso de la Cantera Uruzca no presento rupturas o fragmentaciones que podrían indicar poca dureza en su estructura. Esta condición tiene importancia para la resistencia del hormigón, debido a que parte de esta es dada por la calidad de los agregados que se emplean en el diseño del mismo.

\section{d) Resistividad eléctrica.}

Esta es una propiedad volumétrica del material que se fundamenta en los principios de la Ley de Ohm, en la que se mide el paso de la corriente eléctrica. Este ensayo no destructivo, se relaciona de forma directa con "la resistencia a compresión del hormigón" (Andrade \& D'Andrea, 2011, pp. 91-97) y su vez permite identificar que tan 
susceptible será el hormigón al ataque de agentes externos.

El paso de estos agentes externos mayormente se da por las redes de poros que existen en la estructura interna del hormigón, y mientras más de estas redes se encuentren menor será la resistividad. Investigaciones como (Duffó \& Farina, 2019; Sengul \& Gjorv, 2008) mencionan que la durabilidad del hormigón cuando se ve afectada fuertemente por el paso de agentes químicos, sobre todo en ambientes salinos. A esto se debe añadir que para este tipo de ambientes generalmente se suelen usar cementos con alta impermeabilidad y enfocados para evitar el paso de estos agentes químicos que degradan la armadura de acero y causan perdida de secciones en el hormigón.

Tabla 4: Comparación de resistividad eléctrica y resistencia a la compresión.

\begin{tabular}{cccc}
\hline Días & $\begin{array}{c}\text { Muestra } \\
\left(\mathbf{K g} / \mathbf{c m}^{2}\right)\end{array}$ & $\begin{array}{c}\text { Resistividad } \\
(\mathbf{k} \mathbf{\Omega} . \mathbf{c m})\end{array}$ & $\begin{array}{c}\text { Resistencia a } \\
\text { la } \\
\mathbf{c o m p r e s i o ́ n} \\
\left(\mathbf{K g} / \mathbf{c m}^{2}\right)\end{array}$ \\
\hline \multirow{2}{*}{7} & 210 & 6.75 & 259.15 \\
& 240 & 6.95 & 271.15 \\
28 & 210 & 9.90 & 317.05 \\
& 240 & 9.50 & 351.55 \\
\hline
\end{tabular}

Medidas de resistividad eléctrica obtenidas empleando el equipo SURF'M de GIATEC SCIENTIFIC.
Como se observa la resistividad en las muestras a los 28 días tuvieron un incremento en relación a los primeros 7 días; esto mismo sucedió con la resistencia, aunque la resistividad no dio valores aceptables ante ataque de agentes externos como iones de carbono, cloro o azufre.

La tabla de información que se proporciona en el manual del equipo (Giatec Scientific Inc, s. f.) y basada en la Norma ASTM C1202, indica que una resistividad menor a 10 $\mathrm{k} \Omega . \mathrm{cm}$, tiene una elevada penetración de cloruros. Esto deja marcado que este hormigón no podría ser aplicado en ambientes con alta exposición a iones de carbono, cloro o sulfatos, porque tal como expone (Duffó \& Farina, 2019, pp. 51-64) los criterios de la investigación de Mc Carter et al., 1983 un valor inferior a $10 \mathrm{k} \Omega . \mathrm{cm}$ es sinónimo de altas velocidades de ataque de cloruros o sulfatos al hormigón.

Cuando estos iones de cloro o sulfato de hacen presente en la estructura a elevadas velocidades de penetración lo que disminuye es el tiempo de vida del hormigón; por lo que a pesar de tener elevadas 
resistencias mecánicas a la compresión su durabilidad no será la más adecuada con respecto al tiempo.

Ahora bien, la presencia de estos iones podría generar; primero, la perdida de propiedades mecánicas en el hormigón y el acero, y a su vez una expansión del acero que incrementa el tamaño de grietas dando un mayor acceso a estos agentes químicos y por último una menor durabilidad de la estructura.

\section{Conclusiones}

Los porcentajes de agregado fino que se mezclan para obtener una combinación son de un $80 \%$ cisco lavado de la cantera Uruzca y un $20 \%$ arena de banco del sector de Crucita del cantón Portoviejo. Estos han permitido que se puedan obtener las resistencias adecuadas y superarlas en los tiempos que ha durado la investigación.

Las proporciones que se deben emplear para combinar un saco de cemento con la mezcla de agregados gruesos y finos para una resistencia de $210 \mathrm{~kg} / \mathrm{cm} 2$ son de 1 de cemento con 4.36 de agregados grueso - finos (2.09 de grava pasante tamiz $3 / 4$ in y 2.27 de combinación entre cisco/arena). y para $240 \mathrm{~kg} / \mathrm{cm}^{2}$ es de 1 de cemento con 3.85 de agregados grueso y finos (1.80 de grava pasante tamiz $3 / 4$ in y 2.05 de combinación entre cisco/arena), con estas combinaciones se superan las resistencias esperadas a los 28 días.

La resistencia a la compresión que se alcanzó a los 28 días para el diseño de $210 \mathrm{~kg} / \mathrm{cm}^{2}$ es 315.05 $\mathrm{kg} / \mathrm{cm}^{2}$ y para $240 \mathrm{~kg} / \mathrm{cm}^{2}$ una resistencia de $351.55 \mathrm{~kg} / \mathrm{cm}^{2}$; esto demuestra que los materiales y las proporciones utilizadas para la combinación son correctos para obtener este tipo de resistencia.

Con el ensayo de la resistividad eléctrica se han obtenido valores inferiores a $10 \mathrm{k} \Omega$.cm por lo cual estos hormigones no son actos para ambientes con alta exposición a carbono, cloro o azufre. 


\section{Bibliografía}

Alvarado, S., Vélez, S., Ruiz, W., Ortiz, E., \&

Hernández, E. H. O., Vínces, J. J. G., Castro, C. M. J., \& Zambrano, R. V. H. (2020). Estudio comparativo de resistencias de hormigón de $240 \mathrm{~kg} / \mathrm{cm} 2$ usando los agregados finos, entre el rio Portoviejo y el rio Chone de la Provincia de Manabí. Revista de Investigaciones en Energía, Medio Ambiente $y$ Tecnología. RIEMAT ISSN: 2588-0721, 5(1), 35-44.

Jarre, C. (2019). Estudio de la resistencia a compresión del hormigón utilizando el vidrio finamente molido en reemplazo parcial del cemento. REVISTA RIEMAT, 4(2).

Andrade, C., \& D'Andrea, R. (2011). La resistividad eléctrica como parámetro de control del hormigón y de su durabilidad. Revista ALCONPAT, 1(2), 9098.

https://doi.org/10.21041/ra.v1i 2.8

Duffó, G., \& Farina, S. (2019). Corrosión en estructuras de hormigón armado: Un fenómeno bien conocido pero que aún sigue causando considerables daños. Revista Ciencia e Investigación., 4(60).

https://aargentinapciencias.or g/wp- content/uploads/2019/12/3-

cei69-4-4.pdf

EUCOMEX. (2020). EUCON 37. Reductores de Agua - Alto Rango.

http://www.eucomex.com.mx/ portafolio/productos/aditivos/ $r$ eductores-de-agua-altorango/eucon-37/

Giatec Scientific Inc. (s. f.). User Manual: SURF - Surface resistivity of concrete using wenner four electrode method. Recuperado 16 de enero de 2022, de https://www.giatecscientific.co $\mathrm{m} /$ products/concrete-ndtdevices/surf-surfaceresistivity/

Kosmatka, S., \& Wilson, M. (2011). Design and Controlof Concrete Mixtures: The guide to applications, methods, and materials (Quince). Portland Cement Association.

McCormac, J., \& Brown, R. (2011). Diseño de concreto reforzado (Octava). Alfaomega.

Montejo, A., Montejo, A., \& Montejo, A. (2018). Estabilización de suelos (1.a ed.). Ediciones de la U.

MOP. (2002). Especificaciones Generales para la Construcción de Caminos y Puentes.

NTE INEN 2 380. (2005). Cementos Hidráulicos. Requisitos y desempeño. Instituto 
Ecuatoriano

de

Normalización.

NTE INEN 151. (2010). Cemento hidráulico. Definición de términos. Instituto Ecuatoriano de Normalización.

NTE INEN 156. (2009). Cemento hidráulico. Determinación de la densidad. Instituto Ecuatoriano de Normalización.

NTE INEN 692. (1982). Mecánica de Suelos: Determinación del límite plástico. Instituto Ecuatoriano de Normalización.

NTE INEN 694. (2010). Hormigón y áridos para elaborar hormigones. Terminología. Instituto Ecuatoriano de Normalización.

NTE INEN 696. (2010). Áridos. Análisis granulométrico en los áridos, fino y grueso. Instituto Ecuatoriano de Normalización.

NTE INEN 856. (2010). Áridos. Determinación de la densidad, densidad relativa (Gravedad específica) y absorción del árido fino. Instituto Ecuatoriano de Normalización.

NTE INEN 857. (2010). Áridos. Determinación de la densidad, densidad relativa (Gravedad específica) y absorción del árido grueso.
Instituto Ecuatoriano de Normalización.

NTE INEN 858. (2010). Áridos. Determinación de la masa unitaria (peso volumétrico) y el porcentaje de vacíos. Instituto Ecuatoriano de Normalización.

NTE INEN 862. (2011). Áridos para hormigón. Determinación del contenido total de humedad. Instituto Ecuatoriano de Normalización.

NTE INEN 872. (2011). Áridos para hormigón. Requisitos. Instituto Ecuatoriano de Normalización.

NTE INEN 1573. (2010). Hormigón de cemento hidráulico. Determinación de la resistencia a la compresión de especímenes cilíndricos de hormigón de cemento hidráulico. Instituto Ecuatoriano de Normalización.

NTE INEN 1578. (2010). Hormigón de cemento hidráulico. Determinación del asentamiento. Instituto Ecuatoriano de Normalización.

NTE INEN 2617. (2012). Hormigón de cemento hidráulico. Agua para mezcla. Requisitos. Instituto Ecuatoriano de Normalización.

Panchana, R., Ortiz, E., Ruiz, W., \& Calderero, M. (2020). Fuentes 
de arena de río de la Provincia de Manabí a utilizar en hormigones en la construcción de obras civiles. REVISTA RIEMAT, 5(1).

Parker, H., \& Ambrose, J. (2008). Diseño simplificado de concreto reforzado (3.a ed.). Limusa Wiley.

Quiroz, M., \& Salamanca, L. (2006). Apoyo Didáctico para la enseñanza y aprendizaje en la asignatura de "Tecnología del Hormigón» [Tesis, Universidad Mayor de San Simón]. https://n9.cl/v82x6

Romo, M. (2008). Temas de hormigón armado. Escuela Politécnica del Ejercito.

Sengul, O., \& Gjorv, O. E. (2008). Electrical Resistivity Measurements for Quality Control During Concrete Construction. ACl Materials Journal, 106(6), Article 105M61. https://trid.trb.org/view/87646 5

Soto, I. E. M., \& Escobedo, C. J. M. (2006). Comportamiento mecánico de concreto fabricado con agregados reciclados. Ingeniería. Investigación y Tecnología, VII (3), 151-164.

Yam, J. L. C., Carcaño, R. S., \& Moreno, É. I. (2003). Influencia de los agregados pétreos en las características del concreto. Ingeniería, 7(2), 39-46.

Zabaleta, H. (1992). Compendio de Tecnología del Hormigón. Instituto Chileno del Cemento y del Hormigón. 